International Mathematical Forum, 2, 2007, no. 3, 117 - 123

\title{
On a Dirichlet problem involving p-Laplacian
}

\author{
G.A. Afrouzi \\ Department of Mathematics, Faculty of Basic Sciences, \\ Mazandaran University, Babolsar, Iran \\ e-mail: afrouzi@umz.ac.ir \\ S. Heidarkhani \\ Department of Mathematics, Faculty of Basic Sciences, \\ Mazandaran University, Babolsar, Iran \\ e-mail: s.heidarkhani@umz.ac.ir
}

\begin{abstract}
In this paper, the existence of at least three weak solutions for Dirichlet problem

$$
\begin{cases}\Delta_{p} u+\lambda f(x, u)=0 & \text { in } \Omega, \\ u=0 & \text { on } \partial \Omega,\end{cases}
$$

where $\Delta_{p} u=\operatorname{div}\left(|\nabla u|^{p-2} \nabla u\right)$ is the p-Laplacian operator, $\Omega \subset R^{N}(N \geq$ 1 ) is non-empty bounded open set with smooth boundary $\partial \Omega, p>N$, $\lambda>0$ and $f: \Omega \times R \rightarrow R$ is a $L^{1}$-Caratheodory function, is established. The approach is based on variational methods and critical points.
\end{abstract}

Mathematics Subject Classification: 35J20; 34A15

Keywords: Three solutions; Critical point; Multiplicity results; Dirichlet problem

\section{Introduction}

We consider the boundary value problem

$$
\left\{\begin{array}{lc}
\Delta_{p} u+\lambda f(x, u)=0 & \text { in } \Omega, \\
u=0 & \text { on } \partial \Omega,
\end{array}\right.
$$

where $\Delta_{p} u=\operatorname{div}\left(|\nabla u|^{p-2} \nabla u\right)$ is the p-Laplacian operator, $\Omega \subset R^{N}(N \geq 1)$ is non-empty bounded open set with smooth boundary $\partial \Omega, p>N, \lambda>0$ and 
$f: \Omega \times R \rightarrow R$ is a $L^{1}$-Caratheodory function.

In this paper, under novel assumptions, we are interested in ensuring the existence of at least three weak solutions for the problem (1). As usual, a weak solution of (1) is any $u \in W_{0}^{1, p}(\Omega)$ such that

$$
\int_{\Omega}|\nabla u(x)|^{p-2} \nabla u(x) \nabla v(x) d x-\lambda \int_{\Omega} f(x, u(x)) v(x) d x=0
$$

for all $v \in W_{0}^{1, p}(\Omega)$.

Multiplicity results for problem (1) have been broadly investigated in recent years (see, for example, $[1,2,4,5]$ ); for instance, in [1], using variational methods, the authors ensure the existence of a sequence of arbitrarily small positive solutions for problem (1) when the function $f$ has a suitable oscillating behaviour at zero.

Also, In [4], the author proves multiplicity results for the problem

$$
\left\{\begin{array}{l}
u^{\prime \prime}+\lambda f(u)=0 \\
u(0)=u(1)=0
\end{array}\right.
$$

which for each $\lambda \in\left[0,+\infty\left[\right.\right.$, admits at least three solutions in $W_{0}^{1,2}([0,1])$ when $f$ is a continuous function.

In this paper, Theorem 2.3 which is our main result, under novel assumptions ensures the existence of an open interval $\Lambda \subseteq[0, \infty[$ and a positive real number q such that, for each $\lambda \in \Lambda$, problem (1) admits at least three weak solutions whose norms in $W_{0}^{1, p}(\Omega)$ are less than $\mathrm{q}$.

Our approach is based on a three critical points Theorem proved in [7].

\section{Main Results}

First we recall its equivalent formulation [2, Theorem 1.1 and Remark 1.1] which equivalent with three critical points Theorem:

Theorem 2.1 Let $X$ be a separable and reflexive real Banach space; $\Phi$ : $X \longrightarrow R$ a continuously Gatêaux differentiable and sequentially weakly lower semicontinuous functional whose Gateâux derivative admits a continuous inverse on $X^{*} ; \Psi: X \longrightarrow R$ a continuously Gateâux differentiable functional whose Gatêaux derivative is compact.

Assume that

$$
\lim _{\|u\| \rightarrow+\infty}(\Phi(u)+\lambda \Psi(u))=+\infty
$$

for all $\lambda \in[0,+\infty[$, and that there exists $\rho \in R$ such that

$$
\sup _{\lambda \geq 0} \inf _{u \in X}(\Phi(u)+\lambda \Psi(u)+\rho \lambda)<\inf _{u \in X} \sup _{\lambda \geq 0}(\Phi(u)+\lambda \Psi(u)+\rho \lambda) .
$$


Then, there exists an open interval $\Lambda \subseteq[0,+\infty[$ and a positive real number $q$ such that, for each $\lambda \in \Lambda$, the equation

$$
\Phi^{\prime}(u)+\lambda \Psi^{\prime}(u)=0
$$

has at least three solutions in $X$ whose norms are less than $q$.

Here and in the sequel, $\mathrm{X}$ will denote the Sobolev space $W_{0}^{1, p}(\Omega)$ with the norm

and put

$$
\|u\|=\left(\int_{\Omega}|\nabla u(x)|^{p} d x\right)^{1 / p}
$$

$$
g(x, t)=\int_{0}^{t} f(x, \xi) d \xi
$$

for each $(x, t) \in \Omega \times R$.

Now, fix $x^{0} \in \Omega$ and pick $r_{1}, r_{2}$ with $o<r_{1}<r_{2}$ such that

$$
S\left(x^{0}, r_{1}\right) \subset S\left(x^{0}, r_{2}\right) \subseteq \Omega \text {. }
$$

Put

$$
k_{1}=\frac{1}{r_{2}-r_{1}}\left(\left(r_{2}^{N}-r_{1}^{N}\right) \frac{\pi^{N / 2}}{\Gamma(1+N / 2)}\right)^{1 / p} \mathrm{~cm}(\Omega)^{\frac{1}{N}-\frac{1}{p}}
$$

where $\Gamma$ denotes the Gamma function, $c=c(N, p)$ is a positive constant and $m(\Omega)$ is the Lebesgue measure of the set $\Omega$.

Our main results fully depend on the following Lemma:

Lemma 2.2 Assume that there exist two positive constants $\theta$ and $\tau$ with $k_{1} \tau>\theta$, such that

(i) $g(x, t) \geq 0$ for each $(x, t) \in \Omega \times[0, \tau]$,

(ii) $m(\Omega)\left(k_{1} \tau\right)^{p} \max _{(x, t) \in \bar{\Omega} \times[-\theta, \theta]} g(x, t)<\theta^{p} \int_{S\left(x^{0}, r_{1}\right)} g(x, \tau) d x$,

where $k_{1}$ is given in (3).

Then, there exist $r>0$ and $w \in X$ such that $\|w\|^{p}>p r$ and

$$
m(\Omega) \max g(x, t)<\operatorname{pr} \frac{\int_{\Omega} g(x, w(x)) d x}{\|w\|^{p}}
$$

where $(x, t) \in \bar{\Omega} \times\left[-c|\Omega|^{\frac{1}{N}-\frac{1}{p}} \sqrt[p]{p r}, c|\Omega|^{\frac{1}{N}-\frac{1}{p}} \sqrt[p]{p r}\right]$.

Proof: We put

$$
w(x)= \begin{cases}0 & , x \in \Omega \backslash S\left(x^{0}, r_{2}\right) \\ \frac{\tau}{r_{2}-r_{1}}\left[r_{2}-\sqrt{\sum_{i=1}^{N}\left(x_{i}-x_{i}^{0}\right)^{2}}\right] & , x \in S\left(x^{0}, r_{2}\right) \backslash S\left(x^{0}, r_{1}\right) \\ \tau & , x \in S\left(x^{0}, r_{1}\right)\end{cases}
$$


and $r=\frac{\theta^{p}}{p c^{p} m(\Omega)^{\frac{p}{N}-1}}$. It is easy to see that $w \in X$ and, in particular, one has

$$
\|w\|^{p}=\left(r_{2}^{N}-r_{1}^{N}\right) \frac{\pi^{N / 2}}{\Gamma(1+N / 2)}\left(\frac{\tau}{r_{2}-r_{1}}\right)^{p} .
$$

Hence, taking into account that $k_{1} \tau>\theta$, one has

$$
p r<\|w\|^{p} \text {. }
$$

Since $0 \leq w(x) \leq \tau$ for each $x \in \Omega$, condition (i) ensures that

$$
\int_{S\left(x^{0}, r_{2}\right) \backslash S\left(x^{0}, r_{1}\right)} g(x, w(x)) d x+\int_{\Omega \backslash S\left(x^{0}, r_{2}\right)} g(x, w(x)) d x \geq 0 .
$$

Moreover, owing to our assumptions, we have

$$
m(\Omega) \max g(x, t)<\left(\frac{\theta}{k_{1} \tau}\right)^{p} \int_{S\left(x^{0}, r_{1}\right)} g(x, \tau) d x \leq p r \frac{\int_{\Omega} g(x, w(x)) d x}{\|w\|^{p}}
$$

where $(x, t) \in \bar{\Omega} \times\left[-c|\Omega|^{\frac{1}{N}-\frac{1}{p}} \sqrt[p]{p r}, c|\Omega|^{\frac{1}{N}-\frac{1}{p}} \sqrt[p]{p r}\right]$.

So, the Proof is complete.

Now, we state our main result:

Theorem 2.3 Assume that there exist three positive constants $\theta, \tau, \gamma$ with $k_{1} \tau>\theta, \gamma<p$ and a positive function $a \in L^{1}(\Omega)$ such that

(i) $g(x, t) \geq 0$ for each $(x, t) \in \Omega \times[0, \tau]$,

(ii) $m(\Omega)\left(k_{1} \tau\right)^{p} \max _{(x, t) \in \bar{\Omega} \times[-\theta, \theta]} g(x, t)<\theta^{p} \int_{S\left(x^{0}, r_{1}\right)} g(x, \tau) d x$,

(iii) $g(x, t) \leq a(x)\left(1+|t|^{\gamma}\right)$ almost everywhere in $\Omega$ and for each $t \in R$

where $k_{1}$ is given in (3).

Then, there exists an open interval $\Lambda \subseteq[0,+\infty[$ and a positive real number $q$ such that, for each $\lambda \in \Lambda$, problem (1) admits at least three solutions in $X$ whose norms are less than $q$.

Proof: For each $u \in X$, we put

$\Phi(u)=\frac{\|u\|^{p}}{p}, \quad \Psi(u)=-\int_{\Omega} g(x, u(x)) d x \quad$ and $\quad J(u)=\Phi(u)+\lambda \Psi(u)$.

In particular, for each $u, v \in X$ one has

$$
\begin{gathered}
\Phi^{\prime}(u)(v)=\int_{\Omega}|\nabla u(x)|^{p-2} \nabla u(x) \nabla v(x) d x, \\
\Psi^{\prime}(u)(v)=-\int_{\Omega} f(x, u(x)) v(x) d x .
\end{gathered}
$$


It is well known that the critical points of $J$ are the weak solutions of (1), our goal is to prove that $\Phi$ and $\Psi$ satisfy the assumptions of Theorem 2.1. Clearly, $\Phi$ is a continuously Gateâux differentiable and sequentially weakly lower semi continuous functional whose Gateâux derivative admits a continuous inverse on $X^{*}$ and $\Psi$ is a continuously Gateâux differentiable functional whose Gateâux derivative is compact.

Thanks to (ii), for each $\lambda>0$ one has that

$$
\lim _{\|u\| \rightarrow+\infty}(\Phi(u)+\lambda \Psi(u))=+\infty
$$

We claim that there exist $r>0$ and $w \in X$ such that

$$
\sup _{\left.\left.u \in \Phi^{-1}(]-\infty, r\right]\right)}(-\Psi(u))<r \frac{-(\Psi(w))}{\Phi(w)} .
$$

Now, taking into account that for every $u \in X$, one has

$$
\sup _{x \in \Omega}|u(x)| \leq c m(\Omega)^{\frac{1}{N}-\frac{1}{p}}\|u\|
$$

for each $u \in X$, it follows that

$$
\sup _{\left.\left.u \in \Phi^{-1}(]-\infty, r\right]\right)}(-\Psi(u))=\sup _{\|u\|^{p} \leq p r} \int_{\Omega} g(x, u(x)) d x \leq m(\Omega) \max g(x, t)
$$

where $(x, t) \in \bar{\Omega} \times\left[-c|\Omega|^{\frac{1}{N}-\frac{1}{p}} \sqrt[p]{p r}, c|\Omega|^{\frac{1}{N}-\frac{1}{p}} \sqrt[p]{p r}\right]$.

Now, thanks to Lemma 2.2, there exist $r>0$ and $w \in X$ such that

$$
m(\Omega) \max g(x, t)<p r \frac{\int_{\Omega} g(x, w(x)) d x}{\|w\|^{p}}
$$

where $(x, t) \in \bar{\Omega} \times\left[-c|\Omega|^{\frac{1}{N}-\frac{1}{p}} \sqrt[p]{p r}, c|\Omega|^{\frac{1}{N}-\frac{1}{p}} \sqrt[p]{p r}\right]$.

So

$$
\sup _{\left.\left.u \in \Phi^{-1}(]-\infty, r\right]\right)}(-\Psi(u))<r \frac{-(\Psi(w))}{\Phi(w)} .
$$

Fix $\rho$ such that

$$
\sup _{\left.\left.u \in \Phi^{-1}(]-\infty, r\right]\right)}(-\Psi(u))<\rho<r \frac{-(\Psi(w))}{\Phi(w)}
$$

from Proposition 3.1 of [6], we obtain

$$
\sup _{\lambda \geq 0} \inf _{u \in X}(\Phi(u)+\lambda \Psi(u)+\rho \lambda)<\inf _{u \in X} \sup _{\lambda \geq 0}(\Phi(u)+\lambda \Psi(u)+\rho \lambda) .
$$

Now, our conclusion follows from Theorem 2.1 . 


\section{Two Corollary of Theorem 2.3}

In this section, we put

$$
k_{2}=\frac{1}{r_{2}-r_{1}}\left(\frac{r_{2}^{N}-r_{1}^{N}}{r_{1}^{N}}\right)^{1 / p} \mathrm{~cm}(\Omega)^{\frac{1}{N}-\frac{1}{p}} .
$$

Then, with use Theorem 2.3, we have the following result:

Corollary 3.1 Let $f: R \rightarrow R$ be a continuous function. Put $g(t)=$ $\int_{0}^{t} f(\xi) d \xi$ for each $t \in R$ and assume that there exist four positive constants $\theta$, $\tau, \gamma$ and $\sigma$ with $k_{1} \tau>\theta, \gamma<p$ such that

(j) $g(t) \geq 0$ for each $t \in[0, \tau]$,

$(j j)\left(k_{2} \tau\right)^{p} \max _{t \in[-\theta, \theta]} g(t)<\theta^{p} g(\tau)$,

$(j j j) g(t) \leq \sigma\left(1+|t|^{\gamma}\right)$ for each $t \in R$,

where $k_{1}$ and $k_{2}$ are given in (3) and (4).

Then, there exists an open interval $\Lambda \subseteq[0,+\infty$ [ and a positive real number $q$ such that, for each $\lambda \in \Lambda$, problem

$$
\begin{cases}\Delta_{p} u+\lambda f(u)=0 & \text { in } \Omega, \\ u=0 & \text { on } \partial \Omega,\end{cases}
$$

admits at least three solutions in $X$ whose norms are less than $q$.

Proof: From $(j j)$ and since $\int_{S\left(x^{0}, r_{1}\right)} g(\tau) d x=r_{1}^{N} \frac{\pi^{N / 2}}{\Gamma(1+N / 2)} g(\tau)$, we have

$$
\begin{aligned}
\max _{t \in[-\theta, \theta]} g(t) & <\frac{\theta^{p}}{\left(\frac{\tau}{r_{2}-r_{1}}\right)^{p}\left(r_{2}^{N}-r_{1}^{N}\right) c^{p} m(\Omega)^{\frac{p}{N}-1} \frac{\pi^{N / 2}}{\Gamma(1+N / 2)}} r_{1}^{N} \frac{\pi^{N / 2}}{\Gamma(1+N / 2)} g(\tau) \\
& =\left(\frac{\theta}{k_{1} \tau}\right)^{p} \int_{S\left(x^{0}, r_{1}\right)} g(\tau) d x .
\end{aligned}
$$

Now, our conclusion follows from Theorem 2.3.

If $f: R \rightarrow R$ was a positive continuous function. Then we have:

Corollary 3.2 Assume that there exist four positive constants $\theta, \tau, \gamma$ and $\sigma$ with $k_{1} \tau>\theta, \gamma<p$ such that

$\left(j^{\prime}\right)\left(k_{2} \tau\right)^{p} g(\theta)<\theta^{p} g(\tau)$,

$\left(j j^{\prime}\right) g(t) \leq \sigma\left(1+|t|^{\gamma}\right)$ for each $t \in R$

where $k_{1}$ and $k_{2}$ are given in (3) and (4).

Then, there exists an open interval $\Lambda \subseteq[0,+\infty[$ and a positive real number $q$ such that, for each $\lambda \in \Lambda$, problem (5) admits at least three solutions in $X$ whose norms are less than $q$ 


\section{References}

[1] G. Anello, G. Cordaro, Positive infinitely many arbitrarily small solutions for the Dirichlet problem involving the p-Laplacian, Proc. Roy. Soc. Edinburgh Sect. A, 132 (2002), 511-519.

[2] R.I. Avery, J. Henderson, Three symmetric positive solutions for a secondorder boundary value problem, Appl. Math. lett. 13 (2000), 1-7.

[3] G. Bonanno, A minimax inequality and its applications to ordinary differential equations, J. Math. Anal. Appl. 270 (2002), 210-229.

[4] G. Bonanno, Existence of three solutions for a two point boundary value problem, Appl. Math. Lett. 13 (2000), 53-57.

[5] P. Korman, T. Ouyang, Exact multiplicity results for two classes of boundary value problem, Diff. Integral Equations, 6 (1993), 1507-1517.

[6] B. Ricceri, Existence of three solutions for a class of elliptic eigenvalue problem, Math. Comput. Modelling, 32 (2000), 1485-1494.

[7] B. Ricceri, On a three critical points theorem, Arch. Math. (Basel) 75 (2000), 220-226.

Received: December 14, 2005 